\title{
Immune Checkpoint Inhibitors and Cardiotoxicity: An Analysis of Spontaneous Reports in Eudravigilance
}

\author{
Annamaria Mascolo ${ }^{1,2} \cdot$ Cristina Scavone $^{1,2} \cdot$ Carmen Ferrajolo $^{1,2} \cdot$ Concetta Rafaniello $^{1,2} \cdot$ Romano Danesi $^{3}$. \\ Marzia Del $\mathrm{Re}^{3} \cdot$ Antonio Russo $^{4} \cdot$ Enrico Coscioni $^{5} \cdot$ Francesco Rossi $^{1,2} \cdot$ Roberto Alfano $^{6} \cdot$ Annalisa Capuano $^{1,2}$
}

Accepted: 26 May 2021 / Published online: 18 June 2021

(c) The Author(s) 2021

\begin{abstract}
Introduction Immune checkpoint inhibitors (ICIs) are widely used in the treatment of many cancers as they improve clinical outcomes. However, ICIs have also been associated with the development of immune-related adverse drug reactions (ADRs). Among immune-related ADRs, cardiac immune-related ADRs are rare, but also associated with high mortality rates.

Objective The objective of this study was to evaluate the occurrence of cardiac ADRs reported with ICIs in the European spontaneous reporting system.

Methods We retrieved individual case safety reports on ICI-induced cardiac ADRs from the website of suspected ADR (www.adrreports.eu) of the European pharmacovigilance database (Eudravigilance). Data were retrieved from the date of marketing authorization of each ICI (ipilimumab, nivolumab, pembrolizumab, atezolizumab, durvalumab, avelumab, and cemiplimab) to 14 March, 2020. The reporting odds ratio and its 95\% confidence interval were computed to assess the reporting frequency of cardiac ADRs for each ICI compared to all other ICIs.

Results A total of 2478 individual case safety reports with at least one ICI as the suspected drug were retrieved from Eudravigilance, of which $249(10 \%)$ reported more than one ICI. The three most reported ICIs were nivolumab (43.2\%), pembrolizumab (32.5\%), and the association of nivolumab/ipilimumab (9.4\%). A total of 3388 cardiac ADRs were identified. Cardiac ADRs were serious (99.4\%) and had a fatal outcome (30.1\%). The most reported cardiac events were myocarditis, cardiac failure, atrial fibrillation, pericardial effusion, and myocardial infarction. Nivolumab was reported with a small increased reporting frequency of individual case safety reports with cardiac ADRs compared to all other ICIs (reporting odds ratio $1.09,95 \%$ confidence interval 1.01-1.18).

Conclusions Immune checkpoint inhibitor-induced cardiac ADRs were serious and had unfavorable outcomes. In our study, nivolumab was the only ICI with a small increased reporting frequency of individual case safety reports with cardiac ADRs compared to all other ICIs. In this regard, further head-to-head studies are needed.
\end{abstract}

\section{Introduction}

Immune checkpoint inhibitors (ICIs) are widely used in the treatment of many cancer diseases. Immune checkpoint inhibitors are monoclonal antibodies targeting cytotoxic T-lymphocyte-associated antigen 4 (CTLA-4) or programmed cell death protein 1 or its ligand (PD-1/PD-L1). Cytotoxic T-lymphocyte-associated antigen 4 is a type

Annamaria Mascolo and Cristina Scavone share first authorship.

Roberto Alfano and Annalisa Capuano share lead authorship.

Annamaria Mascolo

annamaria.mascolo@unicampania.it

Extended author information available on the last page of the article

\section{Key Points}

The most reported cardiac events with immune checkpoint inhibitors were myocarditis, cardiac failure, atrial fibrillation, pericardial effusion, and myocardial infarction

Cardiac adverse drug reactions were serious (99.4\%) and had a fatal outcome (30.1\%)

Nivolumab was reported with a small increased reporting frequency of individual case safety reports with cardiac adverse drug reactions compared to all other immune checkpoint inhibitors 
I transmembrane protein expressed on the surface of a $\mathrm{T}$ cell, interacting with two ligands based on the antigen surface: CD80 and CD86. Programmed cell death protein 1 is a T-cell receptor and its binding with PD-L1 and PD-L2 is able to down-modulate the immune system reaction to cancer. Therefore, ICIs allow the re-establishment of the ability of cytotoxic T cells to destroy tumor cells [1]. Currently, seven ICIs obtained the marketing approval: the CTLA-4 inhibitor ipilimumab; PD-1 inhibitors nivolumab, pembrolizumab, and cemiplimab; and the PD-L1 inhibitors, atezolizumab, avelumab, and durvalumab.

Being monoclonal antibodies, ICIs might induce the occurrence of immune-related adverse drug reactions (irADRs) [2]. These type of adverse drug reactions (ADRs) can be the consequence of the effects resulting from $T$ cells acting against antigens shared by tumor and normal cells [3] as well as of the mechanism of action of ICIs. Indeed, molecules targeted by the ICIs are involved in self-tolerance and in autoimmune conditions [4]. Immune checkpoint inhibitorinduced irADRs can involve any tissue and organ and can occur anytime [5]. Among irADRs, cardiac irADRs seem to be rare, but they are associated with a higher mortality rate [6]. These events can involve either the myocardium, the pericardium, or the conduction system [7]. The most frequently reported is myocarditis, even though cases of pericardial disease, Takotsubo cardiomyopathy, and conduction abnormalities have also been reported among ICI users [7]. It is still uncertain if pre-existing risk factors might affect the incidence of ICI-mediated cardiotoxicity [7].

A previous pharmacovigilance study, conducted on data from Vigibase, found that the ICI treatment had a higher reporting of myocarditis and pericardial diseases [8]. However, to our knowledge, no study has investigated the reporting frequency of cardiac ADRs comparing each active ingredient of ICIs. Considering the clinical significance of ICI-related cardiac ADRs, the present pharmacovigilance study is aimed at evaluating the occurrence of cardiac events (in terms of time to onset, seriousness, outcome) after treatment with ICIs and to compare also the reporting frequency of cardiac individual case safety reports (ICSRs) among the drug classes of ICIs through the analysis of data collected in the European spontaneous reporting system.

\section{Methods}

\subsection{Data Source}

Data on ICSRs with an ICI as the suspected drug were retrieved from the website of suspected ADRs (www.adrre ports.eu) of the European pharmacovigilance database (Eudravigilance, EV). The EV is managed by the European Medicines Agency (EMA). It is a system used for the management and analyses of ICSRs related to both medicines or vaccines, which are authorized or are being studied in clinical trials in the European Economic Area. The EV contains all ICSRs reported by a healthcare professional or a non-healthcare professional to an European Union national competent authority or a marketing authorization holder. These data are publicly available for transparency through the EMA website (www.adrreports.eu).

\subsection{ICSR Selection with Line Listing}

By using the line listing function, ICSRs reporting an ICI as the suspected drug and at least one cardiac adverse event were retrieved from the date of marketing authorization granted by the EMA for each ICI to 14 March, 2020. The ICIs considered were ipilimumab, nivolumab, pembrolizumab, atezolizumab, durvalumab, avelumab, and cemiplimab. To identify ICSRs reporting cardiac ADRs, we used the system organ class (SOC) "Cardiac disorders" of the Medical Dictionary for Regulatory Activities (MedDRA). MedDRA is a rich and highly specific standardized medical terminology that facilitates sharing of regulatory information internationally for medical products used by humans. MedDRA is structured into five levels listed hereafter from the most specific to the most general: lowest level terms, preferred terms, high-level terms, high-level group terms, and SOC. In this MedDRA hierarchy, for example, high-level group terms are grouped for etiology (e.g., infections and infestations), manifestation site (e.g., cardiac disorders), or purpose (e.g., surgical and medical procedures) into SOCs.

\subsection{Descriptive Analyses}

Information on patient characteristics (age and sex), adverse event (outcome and seriousness), therapeutic indication, primary source qualification, primary source country for regulatory purposes, number of suspected drugs other than ICIs, and number of concomitant drugs was provided for all ICSRs and separately for each ICI. Individual case safety reports with two or more ICIs as suspected drugs were described separately.

The duration of therapy (reported in days) was retrieved from each ICSR, where available. Computations on the duration of therapy were performed for each ICI, including also data obtained from ICSRs with more ICIs as suspected drugs.

Based on our data source, we have retrieved the general information of seriousness stated as "serious" or "not serious". In accordance with the International Council on Harmonization E2D guidelines, a case is defined as "serious" if it is life threatening, results in death, requires or prolongs a hospitalization, results in persistent or significant disability/ 
incapacity, determines a congenital anomaly/birth defect, or results in some other clinically important conditions. Therefore, if a case is classified as serious in our data source, it means that the case matches one of the aforementioned criteria. However, the specific criteria of seriousness for all ICSRs were not retrievable.

The outcome of a cardiac ADR was classified as "Recovered/Resolved", "Recovering/Resolving", "Recovered/ Resolved with Sequelae", "Not Recovered/Not Resolved", "Fatal", and "Unknown". The outcome with the lower level of resolution was chosen for classification whether an ICSR reported two or more cardiac adverse events with different outcomes. Individual case safety reports were classified as fatal if death occurred.

Cardiac adverse events were tabled for the 20 most reported events in the main table and for all others in the Electronic Supplementary Material (ESM). The time to event (days) was calculated only for ICSRs that reported both the duration of the therapy and the drug withdrawal as action taken after the occurrence of the ADR. Computations on the time to event were performed for each ICI, including also data obtained from ICSRs with more ICIs as suspected drugs. A boxplot of time to event was generated for the most reported ICIs.

To estimate the quota of ICSRs related to patients with pre-existing cardiovascular and metabolic diseases, we evaluated the concomitant presence of cardiovascular and metabolic agents reported as other suspected or concomitant drugs. We listed the most reported drug classes of concomitant treatments (ICSR numbers $\geq 60$ ) in a main table and all others in the ESM. Boxplots were performed using R (Version 3.2.2; R Development Core Team).

\subsection{ROR}

The reporting odds ratio (ROR) and its $95 \%$ confidence interval $(95 \% \mathrm{CI})$ were computed to assess the frequency of reporting ICSRs with ADRs belonging to the SOC "Cardiac disorders" for each ICI (ICI of interest) compared to all other ICIs. The reference group includes all ICIs excluding the one of interest. Moreover, because ICIs can be classified for their mechanism of action in anti-PD-1 (nivolumab, pembrolizumab, and cemiplimab), anti-PD-L1 (atezolizumab, durvalumab, and avelumab), and anti-CTLA-4 (ipilimumab), the ROR was also computed within the same drug class, using all other anti-PD-1 or all other anti-PD-L1 as reference groups, and for comparing anti-CTLA-4 with anti-PD-1/ anti-PD-L1. The RORs were computed on ICSR numbers as these are publicly available on the EMA website (www.adrre ports.eu). Specifically, ICSR numbers were retrieved from the section "Number of individual cases" (www.adrreports. eu) for the period up to 18 March, 2020. Forest plots were performed for both comparisons using R (Version 3.2.2; R Development Core Team).

\section{Results}

\subsection{Characteristics of ICSRs}

During the study period, 2478 ICSRs with at least one ICI as the suspected drug were retrieved from the EV, of which $249(10 \%)$ reported more than one ICI as suspected drugs. Specifically, 1071 (43.2\%) ICSRs pertained to nivolumab, 805 (32.5\%) to pembrolizumab, 234 (9.4\%) to the association of nivolumab/ipilimumab, $143(5.8 \%)$ to ipilimumab, $105(4.2 \%)$ to atezolizumab, 78 (3.1\%) to durvalumab, $23(0.9 \%)$ to avelumab, four $(0.2 \%)$ to cemiplimab, four $(0.2 \%)$ to the association of nivolumab/ pembrolizumab, three $(0.1 \%)$ to nivolumab and atezolizumab, three $(0.1 \%)$ to ipilimumab and pembrolizumab, three $(0.1 \%)$ to atezolizumab and pembrolizumab, and two $(0.1 \%)$ to nivolumab, ipilimumab, and pembrolizumab. The median age of patients was 69 years (interquartile range [IQR] 60.25-75) for all ICSRs (Table 1), remaining similar for all ICI groups except for ICSRs with the association of ipilimumab/pembrolizumab and atezolizumab/ pembrolizumab, in which the median age was 34.5 years (IQR 32.2-36.7) and 49 years (IQR 49-49), respectively (Table 2). Most ICSRs were observed in male patients, and were reported from healthcare professionals (Tables 1,2). Of all ICSRs, $99.4 \%$ were classified as serious. The outcome of cardiac ADRs was "recovered/resolved" in 14.3\% of ICSRs, "recovering/resolving" in $10.9 \%$ of ICSRs, and "Fatal" in $30.1 \%$ of ICSRs (Table 1). In the majority of ICSRs, the ICI was the only suspected drug reported $(n=$ $2179 ; 87.9 \%$ ), or no concomitant drug was reported ( $n=$ $1650 ; 66.6 \%)$. Characteristics of cases for all ICSR groups were presented in Tables 1 and 2.

The main therapeutic indications for all ICIs used as single treatments were lung, genitourinary, and skin cancers (Table 1 of the ESM). For ICSRs reporting more than one ICI as reported in Table 1 of the ESM, the main therapeutic indication for nivolumab/ipilimumab was skin cancer $(N$ $=106 ; 44.9 \%)$, followed by genitourinary cancer $(N=88$; $37.3 \%)$. The median duration of therapy was 43 days for atezolizumab (IQR 2.7-69.2) and durvalumab (IQR 15-88), 30 days (IQR 1-99) for nivolumab, and 22 days for ipilimumab (IQR 1-63.5) and pembrolizumab (IQR 1-66). Because of the scant data available, the median duration of therapy was not computed for avelumab and cemiplimab. 
Table 1 Demographic characteristics and distribution for seriousness, outcomes, primary source, primary source country for regulatory purposes, number of suspected drugs other than ICIs, and number of concomitant drugs of ICSRs reporting at least one cardiac adverse event and having one ICI as the suspected drug among those reported in the Eudravigilance database from the date of marketing authorization to 14 March, 2020

\begin{tabular}{|c|c|c|c|c|c|c|c|c|c|}
\hline Variable & Level & $\begin{array}{l}\text { All ICSRs ( } n \\
=2478)\end{array}$ & $\begin{array}{l}\text { ICSRs with } \\
\text { nivolumab } \\
(n=1071)\end{array}$ & $\begin{array}{l}\text { ICSRs with } \\
\text { pembroli- } \\
\text { zumab }(n= \\
805)\end{array}$ & $\begin{array}{l}\text { ICSRs with } \\
\text { ipilimumab } \\
(n=143)\end{array}$ & $\begin{array}{l}\text { ICSRs with } \\
\text { durvalumab } \\
(n=78)\end{array}$ & $\begin{array}{l}\text { ICSRs with } \\
\text { atezoli- } \\
\text { zumab }(n= \\
105)\end{array}$ & $\begin{array}{l}\text { ICSRs with } \\
\text { avelumab }(n \\
=23)\end{array}$ & $\begin{array}{l}\text { ICSRs with } \\
\text { cemiplimab } \\
(n=4)\end{array}$ \\
\hline Age & $\begin{array}{r}\text { Median } \\
\text { (IQR) }\end{array}$ & $\begin{array}{l}69(60.25- \\
75)\end{array}$ & $69(61-75)$ & $70(62-76)$ & $67(56-75)$ & $71(65-78.5)$ & $\begin{array}{r}65(57- \\
72.25)\end{array}$ & $71(69-76)$ & $\begin{array}{l}70.5(67.7- \\
73.2)\end{array}$ \\
\hline \multirow[t]{3}{*}{ Sex } & Female (\%) & $732(29.5)$ & $314(29.3)$ & $236(29.3)$ & $42(29.4)$ & $20(25.6)$ & $36(34.3)$ & $5(21.7)$ & $1(25.0)$ \\
\hline & Male (\%) & $1652(66.7)$ & 727 (67.9) & $537(66.7)$ & $97(67.8)$ & $51(65.4)$ & $62(59.0)$ & 17 (73.9) & $1(25.0)$ \\
\hline & Missing (\%) & $94(3.8)$ & $30(2.8)$ & $32(4.0)$ & $4(2.8)$ & $7(9.0)$ & $7(6.7)$ & $1(4.3)$ & $2(50.0)$ \\
\hline \multirow{2}{*}{$\begin{array}{l}\text { Seriousness } \\
\text { of ICSR }\end{array}$} & Serious (\%) & 2464 (99.4) & $1062(99.2)$ & 804 (99.9) & $143(100.0)$ & 77 (98.7) & $103(98.1)$ & $22(95.7)$ & $4(100.0)$ \\
\hline & $\begin{array}{l}\text { Not serious } \\
(\%)\end{array}$ & $14(0.6)$ & $9(0.8)$ & $1(0.1)$ & - & $1(1.3)$ & $2(1.9)$ & $1(4.3)$ & - \\
\hline \multirow{6}{*}{$\begin{array}{l}\text { Outcome } \\
\text { of cardiac } \\
\text { event }\end{array}$} & $\begin{array}{l}\text { Recovered/ } \\
\text { resolved }\end{array}$ & $354(14.3)$ & $163(15.2)$ & $121(15.0)$ & $17(11.9)$ & $12(15.4)$ & $12(11.4)$ & $6(26.1)$ & - \\
\hline & $\begin{array}{l}\text { Recovering/ } \\
\text { resolving }\end{array}$ & $271(10.9)$ & $110(10.3)$ & $98(12.2)$ & $13(9.1)$ & $7(9.0)$ & $13(12.4)$ & $2(8.7)$ & - \\
\hline & $\begin{array}{l}\text { Recovered } \\
\text { with } \\
\text { sequelae }\end{array}$ & $25(1.0)$ & $12(1.1)$ & $10(1.2)$ & $1(0.7)$ & - & $1(0.9)$ & - & - \\
\hline & $\begin{array}{l}\text { Not recov- } \\
\text { ered/not } \\
\text { resolved }\end{array}$ & $177(7.1)$ & $67(6.3)$ & $71(8.8)$ & $7(4.9)$ & $2(2.6)$ & $10(9.5)$ & $3(13.0)$ & $2(50.0)$ \\
\hline & Fatal & $745(30.1)$ & 297 (27.7) & $262(32.5)$ & $51(35.7)$ & $25(32.1)$ & $26(24.8)$ & $8(34.8)$ & - \\
\hline & Unknown & 906 (36.6) & $422(39.4)$ & $243(30.2)$ & $54(37.8)$ & $32(41.0)$ & $43(41.0)$ & $4(17.4)$ & $2(50.0)$ \\
\hline \multirow[t]{2}{*}{$\begin{array}{r}\text { Primary } \\
\text { source }\end{array}$} & $\begin{array}{l}\text { Healthcare } \\
\text { profes- } \\
\text { sional }\end{array}$ & 2317 (93.5) & 975 (91.0) & 780 (96.9) & $124(86.7)$ & 77 (98.7) & $103(98.1)$ & $23(100.0)$ & $4(100.0)$ \\
\hline & $\begin{array}{l}\text { Non-health- } \\
\text { care pro- } \\
\text { fessional }\end{array}$ & $161(6.5)$ & $96(9.0)$ & $25(3.1)$ & $19(13.3)$ & $1(1.3)$ & $2(1.9)$ & - & - \\
\hline \multirow{2}{*}{$\begin{array}{l}\text { Primary } \\
\text { source } \\
\text { country for } \\
\text { regulatory } \\
\text { purposes }\end{array}$} & $\begin{array}{l}\text { European } \\
\text { economic } \\
\text { area }\end{array}$ & $934(37.7)$ & $481(44.9)$ & $256(31.8)$ & $53(37.1)$ & $18(23.1)$ & $41(39.0)$ & $14(60.9)$ & $1(25.0)$ \\
\hline & $\begin{array}{l}\text { Non- } \\
\text { European } \\
\text { economic } \\
\text { area }\end{array}$ & $1,544(62.3)$ & $590(55.1)$ & $549(68.2)$ & $90(62.9)$ & $60(76.9)$ & $64(61.0)$ & $9(39.1)$ & $3(75.0)$ \\
\hline \multirow{6}{*}{$\begin{array}{l}\text { Suspected } \\
\text { drug(s) } \\
\text { other than } \\
\text { ICIs }\end{array}$} & 0 & $2,179(87.9)$ & 958 (89.4) & $699(86.8)$ & 134 (93.7) & $65(83.3)$ & $78(74.3)$ & $12(52.2)$ & $4(100.0)$ \\
\hline & 1 & $163(6.6)$ & $78(7.3)$ & $42(5.2)$ & $6(4.2)$ & $5(6.4)$ & $15(14.3)$ & $4(17.4)$ & - \\
\hline & 2 & $93(3.7)$ & $23(2.1)$ & $51(6.3)$ & $2(1.4)$ & $6(7.7)$ & $5(4.8)$ & $3(13.0)$ & - \\
\hline & 3 & $21(0.8)$ & $8(0.7)$ & $4(0.5)$ & $1(0.7)$ & $1(1.3)$ & $4(3.8)$ & $2(8.7)$ & - \\
\hline & 4 & $12(0.5)$ & $2(0.2)$ & $5(0.6)$ & - & $1(1.3)$ & $2(1.9)$ & $1(4.3)$ & - \\
\hline & $\geq 5$ & $10(0.4)$ & $2(0.2)$ & $4(0.5)$ & - & - & $1(0.9)$ & $1(4.3)$ & - \\
\hline \multirow{6}{*}{$\begin{array}{c}\text { Concomitant } \\
\operatorname{drug}(\mathrm{s})\end{array}$} & 0 & $1,650(66.6)$ & $699(65.3)$ & $522(64.8)$ & $88(61.5)$ & $56(71.8)$ & $82(78.1)$ & $14(60.9)$ & $3(75.0)$ \\
\hline & 1 & $145(5.8)$ & $73(6.8)$ & 37 (4.6) & $12(8.4)$ & $4(5.1)$ & $6(5.7)$ & - & $1(25.0)$ \\
\hline & 2 & $129(5.2)$ & $53(4.9)$ & $53(6.6)$ & $5(3.5)$ & $1(1.3)$ & $9(8.6)$ & $1(4.3)$ & - \\
\hline & 3 & $79(3.2)$ & $42(3.9)$ & $26(3.2)$ & $4(2.8)$ & $1(1.3)$ & $1(0.9)$ & - & - \\
\hline & 4 & $80(3.2)$ & $38(3.5)$ & $24(3.0)$ & $8(5.6)$ & $1(1.3)$ & $1(0.9)$ & $2(8.7)$ & - \\
\hline & $\geq 5$ & $395(15.9)$ & $166(15.5)$ & $143(17.8)$ & $26(18.2)$ & 15 (19.2) & $6(5.7)$ & $6(26.1)$ & - \\
\hline
\end{tabular}

ICIs immune checkpoint inhibitors, ICSRs individual case safety reports, IQR interquartile range 
Table 2 Demographic characteristics and distribution for seriousness, outcomes, primary source, primary source country for regulatory purposes, number of suspected drugs other than ICIs, and number of concomitant drugs of ICSRs reporting at least one cardiac adverse

\begin{tabular}{|c|c|c|c|c|c|c|c|}
\hline Variable & Level & $\begin{array}{l}\text { ICSRs with } \\
\text { nivolumab and } \\
\text { ipilimumab ( } \\
=234 \text { ) }\end{array}$ & $\begin{array}{l}\text { ICSRs with } \\
\text { nivolumab and } \\
\text { pembrolizumab } \\
(n=4)\end{array}$ & $\begin{array}{l}\text { ICSRs with } \\
\text { nivolumab and } \\
\text { atezolizumab ( } n \\
=3 \text { ) }\end{array}$ & $\begin{array}{l}\text { ICSRs with } \\
\text { nivolumab, } \\
\text { ipilimumab, and } \\
\text { pembrolizumab } \\
(n=2)\end{array}$ & $\begin{array}{l}\text { ICSRs with } \\
\text { ipilimumab and } \\
\text { pembrolizumab } \\
(n=3)\end{array}$ & $\begin{array}{l}\text { ICSRs with } \\
\text { atezolizumab and } \\
\text { pembrolizumab } \\
(n=3)\end{array}$ \\
\hline Age & Median (IQR) & $66(55-71)$ & $69(58.5-73.5)$ & $64(65.5-62.5)$ & - & $\begin{array}{l}34.5(32.2- \\
36.7)\end{array}$ & 49 (49-49) \\
\hline \multirow[t]{3}{*}{ Sex } & Female (\%) & $73(31.2)$ & $2(50.0)$ & $2(66.7)$ & - & - & - \\
\hline & Male (\%) & $150(64.1)$ & $2(50.0)$ & $1(33.3)$ & $2(100.0)$ & $3(100.0)$ & $3(100.0)$ \\
\hline & Missing (\%) & $11(4.7)$ & - & - & - & - & - \\
\hline \multirow[t]{2}{*}{ Seriousness } & Serious (\%) & $234(100.0)$ & $4(100.0)$ & $3(100.0)$ & $2(100.0)$ & $3(100.0)$ & $3(100.0)$ \\
\hline & Not serious (\%) & - & - & - & - & - & - \\
\hline \multirow[t]{6}{*}{ Outcome } & $\begin{array}{l}\text { Recovered/ } \\
\text { resolved }\end{array}$ & $20(8.5)$ & - & $1(33.3)$ & $2(100.0)$ & - & - \\
\hline & $\begin{array}{l}\text { Recovering/ } \\
\text { resolving }\end{array}$ & $26(11.1)$ & $1(25.0)$ & - & - & - & $1(33.3)$ \\
\hline & $\begin{array}{l}\text { Recovered with } \\
\text { sequelae }\end{array}$ & - & - & - & - & $1(33.3)$ & - \\
\hline & $\begin{array}{c}\text { Not recovered/ } \\
\text { not resolved }\end{array}$ & $15(6.4)$ & - & - & - & - & - \\
\hline & Fatal & $71(30.3)$ & $2(50.0)$ & $2(66.7)$ & - & $1(33.3)$ & - \\
\hline & Unknown & $102(43.6)$ & $1(25.0)$ & - & - & $1(33.3)$ & $2(66.7)$ \\
\hline \multirow[t]{2}{*}{ Primary source } & $\begin{array}{l}\text { Healthcare } \\
\text { professional }\end{array}$ & $216(92.3)$ & $4(100.0)$ & $3(100.0)$ & $2(100.0)$ & $3(100.0)$ & $3(100.0)$ \\
\hline & $\begin{array}{l}\text { Non-healthcare } \\
\text { professional }\end{array}$ & $18(7.7)$ & - & - & - & - & - \\
\hline \multirow{2}{*}{$\begin{array}{l}\text { Primary source } \\
\text { country for } \\
\text { regulatory } \\
\text { purposes }\end{array}$} & $\begin{array}{c}\text { European eco- } \\
\text { nomic area }\end{array}$ & 67 (28.6) & $1(25.0)$ & $1(33.3)$ & - & $1(33.3)$ & - \\
\hline & $\begin{array}{l}\text { Non-European } \\
\text { economic area }\end{array}$ & 167 (71.4) & $3(75.0)$ & $2(66.7)$ & $2(100.0)$ & $2(66.7)$ & $3(100.0)$ \\
\hline \multirow{6}{*}{$\begin{array}{l}\text { Suspected } \\
\text { drug(s) other } \\
\text { than ICIs }\end{array}$} & 0 & 215 (91.9) & $3(75.0)$ & $3(100.0)$ & $2(100.0)$ & $3(100.0)$ & $3(100.0)$ \\
\hline & 1 & $13(5.6)$ & - & - & - & - & - \\
\hline & 2 & $3(1.3)$ & - & - & - & - & - \\
\hline & 3 & $1(0.4)$ & - & - & - & - & - \\
\hline & 4 & $1(0.4)$ & - & - & - & - & - \\
\hline & $\geq 5$ & $1(0.4)$ & $1(25.0)$ & - & - & - & - \\
\hline \multirow{6}{*}{$\begin{array}{l}\text { Concomitant } \\
\operatorname{drug}(\mathrm{s})\end{array}$} & 0 & $178(76.1)$ & - & $2(66.7)$ & - & $3(100.0)$ & $3(100.0)$ \\
\hline & 1 & $12(5.1)$ & - & - & - & - & - \\
\hline & 2 & $6(2.6)$ & $1(25.0)$ & - & - & - & - \\
\hline & 3 & $5(2.1)$ & - & - & - & - & - \\
\hline & 4 & $3(1.3)$ & $2(50.0)$ & $1(33.3)$ & - & - & - \\
\hline & $\geq 5$ & $30(12.8)$ & $1(25.0)$ & - & $2(100.0)$ & - & - \\
\hline
\end{tabular}

ICIs immune checkpoint inhibitors, ICSRs individual case safety reports, IQR interquartile range

\subsection{Cardiac Adverse Events}

We observed a total of 3078 cardiac ADRs (1.2 cardiac ADRs per ICSR), as more than one ADR could be reported in each ICSR. A total of 1310 (42.6\%) cardiac ADRs were

event and having two or more ICIs as suspected drugs among those reported in the Eudravigilance database from the date of marketing authorization to 14 March, 2020

$4(65.5-62.5)-$

$34.5(32.2-\quad 49(49-49)$

$(66.7)$

ipilimumab $(n$ pembrolizumab 政 . 
four $(0.1 \%)$ with nivolumab/pembrolizumab, four $(0.1 \%)$ with nivolumab/ipilimumab/pembrolizumab, three $(0.1 \%)$ with ipilimumab/pembrolizumab, and three $(0.1 \%)$ with nivolumab/atezolizumab. Overall, the most reported cardiac adverse events were myocarditis, cardiac failure, atrial fibrillation, pericardial effusion, and myocardial infarction. Numbers and percentages of cardiac adverse events are reported in Tables 3 and 4, and Table 2 of the ESM. The number of ICSRs for which we were able to calculate the time to event were: 188 for nivolumab, 144 for pembrolizumab, 39 for ipilimumab, 18 for durvalumab, nine for atezolizumab, two for avelumab, and one for cemiplimab. The median time to event was 36 days for atezolizumab (IQR 2-57) and durvalumab (IQR 15-81.75), 31 days (IQR 1.75-96.75) for nivolumab, and 22 days for pembrolizumab (IQR 1-64) and ipilimumab (IQR 1-36.5). Because of the scant data, the time to event was not computed for avelumab and cemiplimab. Figure 1 shows the boxplots of times to event for the most reported ICIs.

\subsection{Cardiovascular and Metabolic Agents Identified in the ICSRs}

In 57 (2.3\%) out of 2478 ICSRs, cardiovascular and metabolic agents were reported among suspected drugs. Specifically, 25 out of 57 ICSRs (43.9\%) were related to nivolumab, $18(31.6 \%)$ to pembrolizumab, four (7.0\%) to atezolizumab, four $(7.0 \%)$ to the association of nivolumab/ipilimumab, three (5.3\%) to ipilimumab, two (3.5\%) to durvalumab, and one $(1.7 \%)$ to avelumab. A total of 78 cardiovascular and metabolic agents were identified in the ICSRs. The most reported were calcium channel blockers (ten; $12.8 \%$ ), new oral anticoagulants (ten; $12.8 \%$ ), statins (nine; 11.5\%), betablockers (nine; 11.5\%), and loop diuretics (seven; 9.0\%). Cardiovascular and metabolic agents reported as other suspected drugs are shown in Table 5.

In 543 (21.9\%) out of 2478 ICSRs, cardiovascular and metabolic agents were reported among concomitant drugs. Specifically, 259 out of 543 ICSRs (47.7\%) were referred to nivolumab, $176(32.4 \%)$ to pembrolizumab, 33 (6.1\%) to ipilimumab, $31(5.7 \%)$ to the association of nivolumab/ ipilimumab, $21(3.9 \%)$ to durvalumab, nine (1.6\%) to atezolizumab, seven $(1.3 \%)$ to avelumab, three $(0.5 \%)$ to the association of nivolumab/pembrolizumab, two (0.4\%) to the association of nivolumab/ipilimumab/pembrolizumab, one $(0.2 \%)$ to the association of nivolumab/atezolizumab, and one $(0.2 \%)$ to cemiplimab. A total of 1330 concomitant drugs were identified in the ICSRs. The most reported were beta-blockers $(172 ; 12.9 \%)$, calcium channel blockers $(145 ; 10.9 \%)$, antiplatelet agents $(142 ; 10.7 \%)$, statins $(139 ; 10.5 \%)$, and angiotensin receptor blockers $(92 ; 6.9 \%)$ (Table 6). Details of cardiovascular and metabolic agents reported as concomitant drugs are shown in Table 3 of the ESM.

\subsection{Comparison of the Reporting Frequency of ICSRs with Cardiac ADRs Among ICls}

Compared to all other ICIs, nivolumab reported a very small increased reporting frequency of ICSRs with cardiac ADRs (ROR 1.09, 95\% CI 1.01-1.18). Moreover, anti-CTLA-4 has shown a lower reporting frequency of ICSRs with cardiac ADRs compared to anti-PD-1 (ROR 0.75, 95\% CI 0.67-0. 84 ) and anti-PD-L1 (ROR 0.79, 95\% CI 0.66-0.93). All computations are reported in Figs. 2, 3, 4. The numbers of ICI reports used for the ROR computations are shown in Table 4 of the ESM.

\section{Discussion}

\subsection{Overall Results}

The present study evaluated the reporting of cardiac ICSRs with ICIs using data from the EV database. Data reported in the EV database concerns suspected side effects that have been observed following the use of a medicine, but that are not necessarily related to or caused by the medicine. We found that the main suspected ICIs were nivolumab and pembrolizumab and, overall, the main cardiac events were myocarditis, cardiac failure, atrial fibrillation, pericardial effusion, and myocardial infarction. Ninety-nine percent of cardiac ADRs was classified as serious and $30.1 \%$ had a fatal outcome. Among ICIs, nivolumab reported a small increased reporting frequency of ICSRs with cardiac ADRs compared to all other ICIs.

In our study, more than $76 \%$ of all ICSRs $(n=1876)$ were related to nivolumab and pembrolizumab, while the remaining ICSRs reported as suspected other ICIs or their associations. In our opinion, the higher number of ICSRs reported with nivolumab and pembrolizumab might depend on their earlier marketing approval. Indeed, together with ipilimumab, which was the first authorized CTLA-4 inhibitor, nivolumab and pembrolizumab were the first PD-Lis to be approved [9].

In the present analysis, the majority of ADRs with ICIs was reported in male patients with a median age of 69 years. For all ICIs, the main therapeutic indications were lung, genitourinary, and skin cancers. These data are not surprising if we consider that ICIs are mainly indicated for the treatment of melanoma, renal cell carcinoma, bladder cancer, head and neck cancers, and lung cancers [10]. These conditions show higher prevalences in male adult patients. For instance, melanoma incidence reaches a peak at the seventh and eighth decades of life and, after the age of 40 years, it affects more 


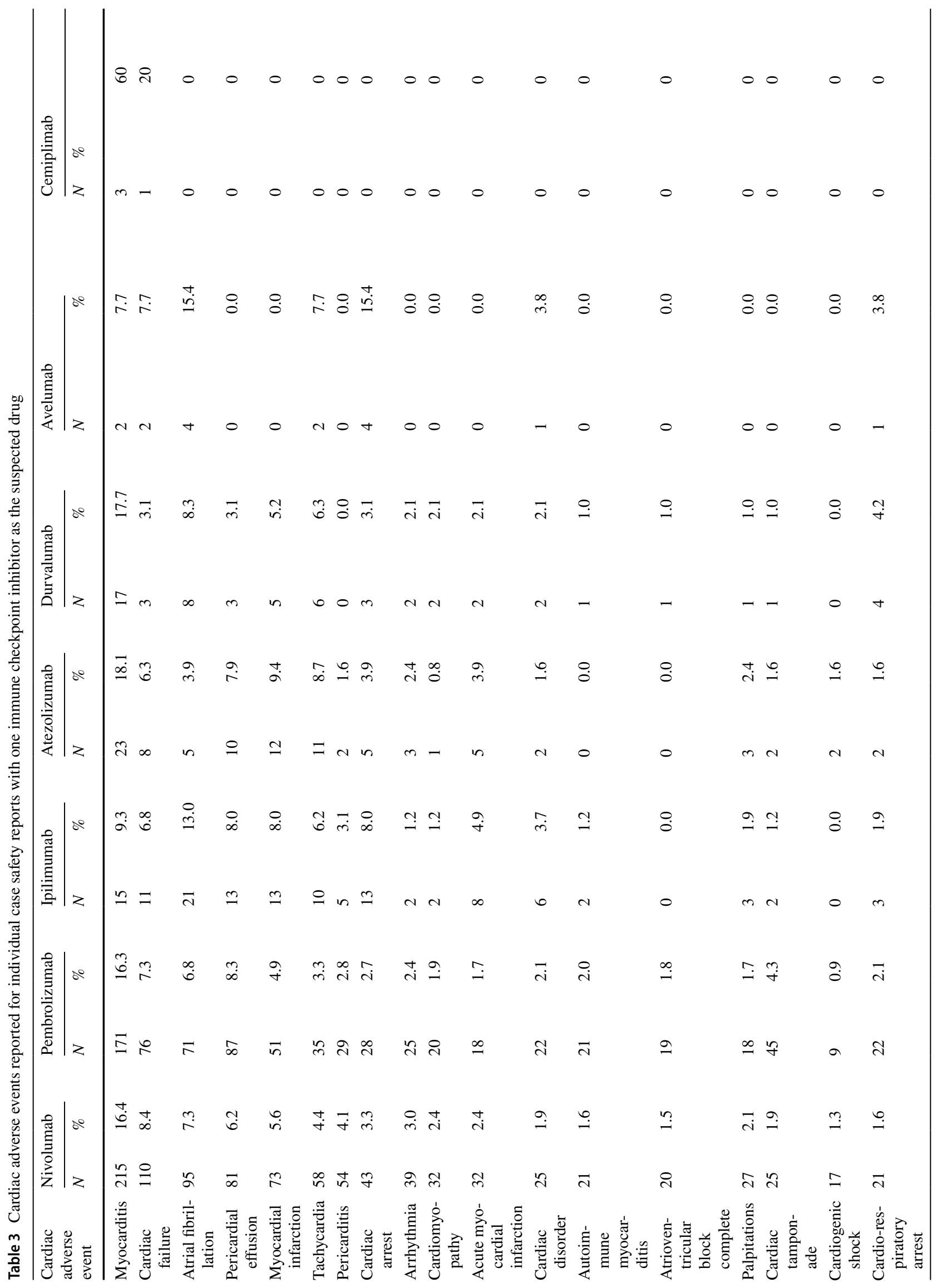




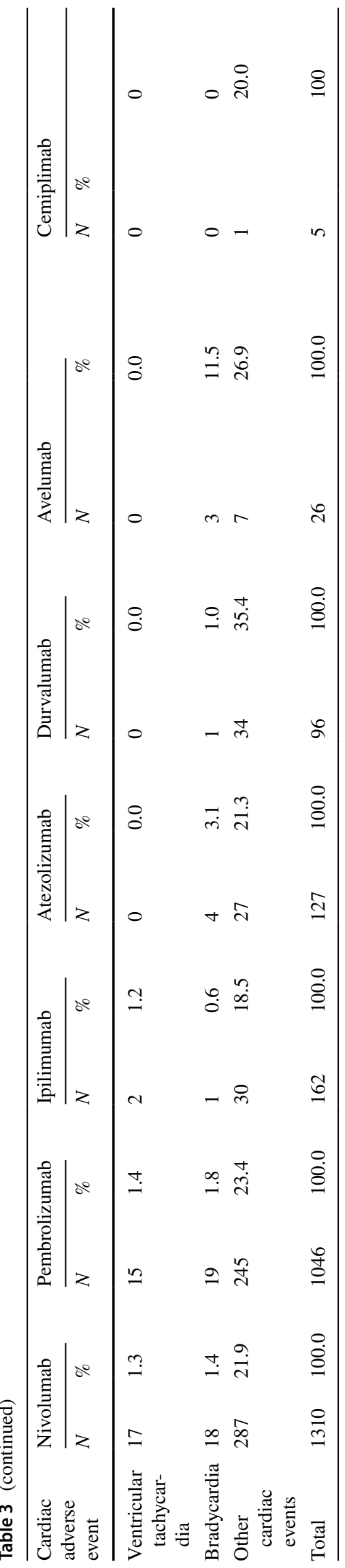

men than women [11]. Similar trends and risk factors were observed for other cancers, including renal cell carcinoma [12] and lung cancers [13]. Last, the higher proportion of ADRs in male patients was previously found for all ICIs in a pharmacovigilance study that used data from the Italian spontaneous reporting system [14]. We also found that combinations of ipilimumab/pembrolizumab and atezolizumab/ pembrolizumab were commonly reported in patients with a median age of 34.5 years and 49 years, respectively. These combinations are increasingly used in patients with metastatic melanoma, advanced renal cell carcinoma, and colorectal and lung cancer owing to their effect in improving the response rates and median survival time [15].

Most of the cases were serious. Taking into account that ICIs are monoclonal antibodies, these results are not surprising. Monoclonal antibodies have longer terminal half-lives and consequently even a single dose of these drugs leads to prolonged systemic exposure that increases the risk of serious adverse events [16]. Moreover, in the evaluation of seriousness, we should also consider the reporting bias of serious ADRs due to mandatory reporting obligations by regulatory authorities and that healthcare professionals are more prone to report serious than non-serious ADRs.

Almost $30 \%$ of ICSRs reported ADRs with a fatal outcome, while approximately $25 \%$ reported ADRs with a favorable outcome. It is well known that ICIs are able to induce many types of irADRs affecting the colon, liver, lungs, thyroid, skin, heart, and nervous system. Even though irADRs are usually manageable with corticosteroids or immune modulator agents, uncommon fatal events have been reported [17]. However, an important consideration that should be stated in regard to suspected adverse events retrieved from spontaneous reporting systems, with greater attention for those that are lethal, is that we cannot be sure of their causal relationship with a drug. Indeed, in the spontaneous reporting system are reported adverse events observed during a drug treatment but that are not obligatorily related to it. Therefore, their onset and severity could depend on other factors such as the frailty of the population, the nature of their underlying illness, or even to the specific reporting practices of those sending the ICSRs for this type of patient. In this study, the median duration of ICI therapy had a range of 22-43 days. In our opinion, many reasons could explain these data, including the withdrawal of the drug as an action taken after the occurrence of cardiac ADRs, the seriousness of some cancer diseases, such as lung cancer, which are associated with a high mortality rate in the short term, and last, the lack of a follow-up for ICSRs retrieved from the EV database. 
Table 4 Cardiac adverse events reported for individual case safety reports with more immune checkpoint inhibitors as suspected drugs

\begin{tabular}{|c|c|c|c|c|c|c|c|c|c|c|c|}
\hline \multirow[t]{2}{*}{ Cardiac adverse event } & \multicolumn{2}{|c|}{$\begin{array}{l}\text { Nivolumab } \\
\text { and ipili- } \\
\text { mumab }\end{array}$} & \multicolumn{6}{|c|}{$\begin{array}{l}\text { Nivolumab Ipilimumab Nivolumab } \\
\text { and pem- and pem- and atezoli- } \\
\text { brolizumab brolizumab zumab }\end{array}$} & \multicolumn{2}{|c|}{$\begin{array}{l}\text { Atezoli- } \\
\text {-zumab } \\
\text { and } \\
\text { pem- } \\
\text { broli- } \\
\text { zumab }\end{array}$} & $\begin{array}{l}\text { Nivolumab, } \\
\text { ipilimumab, } \\
\text { and pembroli- } \\
\text { zumab }\end{array}$ \\
\hline & $N$ & $\%$ & $N$ & $\%$ & $N$ & $\%$ & $N$ & $\%$ & $N$ & $\%$ & $\%$ \\
\hline Myocarditis & 91 & 31.7 & 0 & 0.0 & 2 & 66.7 & 3 & 100.0 & 0 & 0.02 & 50.0 \\
\hline Cardiac failure & 23 & 8.0 & 1 & 25.0 & 0 & 0.0 & 0 & 0.0 & 0 & 0.00 & 0.0 \\
\hline Atrial fibrillation & 17 & 5.9 & 0 & 0.0 & 0 & 0.0 & 0 & 0.0 & 0 & 0.00 & 0.0 \\
\hline Pericardial effusion & 8 & 2.8 & 1 & 25.0 & 0 & 0.0 & 0 & 0.0 & 2 & 40.00 & 0.0 \\
\hline Myocardial infarction & 12 & 4.2 & 0 & 0.0 & 0 & 0.0 & 0 & 0.0 & 0 & 0.00 & 0.0 \\
\hline Tachycardia & 13 & 4.5 & 1 & 25.0 & 0 & 0.0 & 0 & 0.0 & 0 & 0.00 & 0.0 \\
\hline Pericarditis & 3 & 1.0 & 0 & 0.0 & 0 & 0.0 & 0 & 0.0 & 0 & 0.00 & 0.0 \\
\hline Cardiac arrest & 11 & 3.8 & 0 & 0.0 & 0 & 0.0 & 0 & 0.0 & 0 & 0.00 & 0.0 \\
\hline Arrhythmia & 9 & 3.1 & 0 & 0.0 & 0 & 0.0 & 0 & 0.0 & 0 & 0.00 & 0.0 \\
\hline Cardiomyopathy & 6 & 2.1 & 0 & 0.0 & 1 & 33.3 & 0 & 0.0 & 0 & 0.00 & 0.0 \\
\hline Acute myocardial infarction & 1 & 0.3 & 0 & 0.0 & 0 & 0.0 & 0 & 0.0 & 0 & 0.00 & 0.0 \\
\hline Cardiac disorder & 6 & 2.1 & 0 & 0.0 & 0 & 0.0 & 0 & 0.0 & 0 & 0.00 & 0.0 \\
\hline Autoimmune myocarditis & 7 & 2.4 & 1 & 25.0 & 0 & 0.0 & 0 & 0.0 & 0 & 0.00 & 0.0 \\
\hline $\begin{array}{l}\text { Atrioventricular block com- } \\
\text { plete }\end{array}$ & 5 & 1.7 & 0 & 0.0 & 0 & 0.0 & 0 & 0.0 & 0 & 0.02 & 50.0 \\
\hline Palpitations & 0 & 0.0 & 0 & 0.0 & 0 & 0.0 & 0 & 0.0 & 0 & 0.00 & 0.0 \\
\hline Cardiac tamponade & 1 & 0.3 & 0 & 0.0 & 0 & 0.0 & 0 & 0.0 & 0 & 0.00 & 0.0 \\
\hline Cardiogenic shock & 9 & 3.1 & 0 & 0.0 & 0 & 0.0 & 0 & 0.0 & 0 & 0.00 & 0.0 \\
\hline Cardio-respiratory arrest & 5 & 1.7 & 0 & 0.0 & 0 & 0.0 & 0 & 0.0 & 0 & 0.00 & 0.0 \\
\hline Ventricular tachycardia & 5 & 1.7 & 0 & 0.0 & 0 & 0.0 & 0 & 0.0 & 0 & 0.00 & 0.0 \\
\hline Bradycardia & 2 & 0.7 & 0 & 0.0 & 0 & 0.0 & 0 & 0.0 & 0 & 0.00 & 0.0 \\
\hline Other cardiac events & 53 & 18.5 & 0 & 0.0 & 0 & 0.0 & 0 & 0.0 & 3 & 60.00 & 0.0 \\
\hline Total & 287 & 100.0 & 4 & 100.0 & 3 & 100.0 & 3 & 100.0 & 5 & 100.04 & 100.0 \\
\hline
\end{tabular}

\subsection{Cardiac Adverse Events}

As previously reported, in our study, the main identified cardiac events were myocarditis, cardiac failure, atrial fibrillation, pericardial effusion, and myocardial infarction. Many hypotheses have been formulated with regard to the association between ICIs and cardiotoxicity. Indeed, Freeman et al. reported that PD-L1 might regulate autoreactive lymphocytes playing a role in limiting activities of $\mathrm{T}$ cells in the heart, where PD-L1 is highly expressed [37]. It was also demonstrated that the disruption of the gene encoding for PD-1 in mice caused dilated cardiomyopathy [18]. In addition, hyperactivated CD4+/CD8+ T lymphocytes and macrophages could be microscopically found in the heart tissue of patients with ICI-related cardiac toxicity. One of the most likely explanations underlying the occurrence of this toxicity is the "shared antigen" hypothesis between the tumor and cardiac muscle, which could be related to muscle-specific antigens, desmin and troponin, detected in the tumor. Thus, a response to cardiac antigens might contribute to heart failure through an autoantibody-independent mechanism [19]. Among patients treated with ICIs, up to 60-80\% experience at least one irADR and the incidence of cardiac irADRs has a range of 1.14-5\%.

In line with literature data, in our study, myocarditis was the most commonly reported cardiac ADR. However, other signs and symptoms of cardiotoxicity could occur, including arrhythmias, atrial fibrillation, conduction disorders, pericardial disease, and vasculitis [20]. All these ADRs were found to be possibly related to ICIs in our study too. In our study, the majority of cardiac events were reported for nivolumab, followed by pembrolizumab, ipilimumab, and atezolizumab. In terms of the distribution of cardiac ADRs by suspected ICIs, some differences were found. Indeed, in line with literature data suggesting that the combination ICI therapy (e.g., a CTLA-4 inhibitor combined with a PD-1 inhibitor) is the predominant risk factor for myocarditis and that pericardial diseases occur more frequently in patients receiving antiPD-1 or anti-PDL-1 antibodies than those treated with antiCTLA-4 [21], we found that almost $30 \%$ of cardiac ADRs related to the combination nivolumab/ipilimumab concerned cases of myocarditis, while ADRs affecting the pericardium 
Fig. 1 Time to the event of individual case safety reports (ICSRs) with ipilimumab, nivolumab, and pembrolizumab. $I Q R$ interquartile range

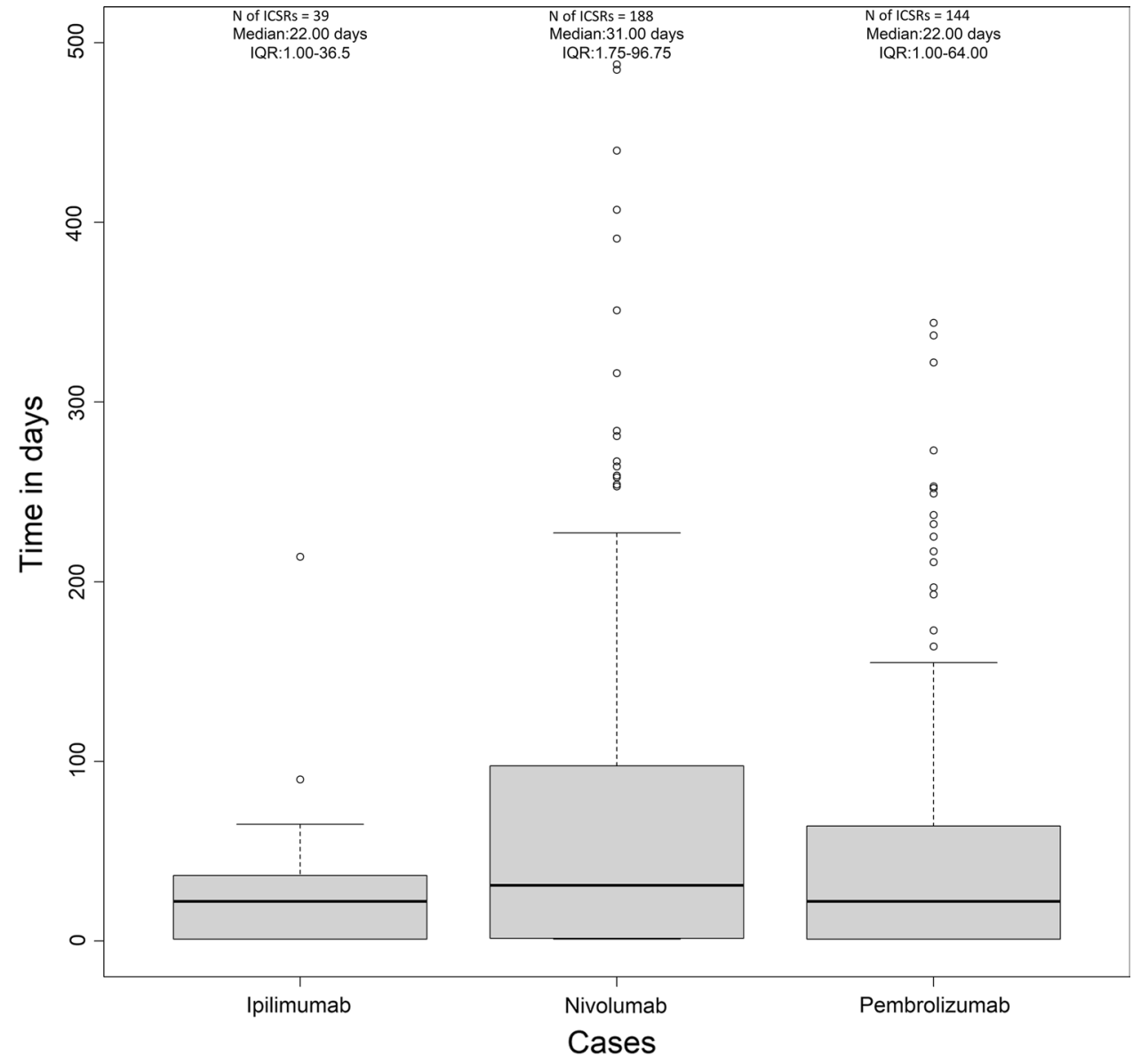

were rarely reported with ipilimumab alone compared to other ICIs.

In terms of the disproportionality analysis, we observed a very small increased reporting of cardiac events with nivolumab (9\% higher frequency of reporting ICSRs than the other ICI). This result may reflect the higher number/ proportion of ICSRs on nivolumab present in the database, considering also that nivolumab is approved for diverse cancer types and has been in use for a longer time compared to anti-PD-L1 ICIs. Among ICIs within the same drug class (anti-PD-1 or anti-PD-L1), no difference for the reporting frequency of cardiac ICSRs was found, while anti-CTLA-4 had a lower reporting of cardiac ICSRs compared with antiPD-1 and anti-PD-L1.

On the contrary, literature data suggest that cardiac irADRs seem to be more common in patients treated with the CTLA-4 inhibitor compared with those receiving antiPD-1. The risk increases with combination therapy, leading to a discontinuation rate in up to $50 \%$ of treated patients [22]. It is important to highlight that we performed a disproportionality analysis with RORs restricted to the ICI drug class for assessing the reporting frequency of cardiac ICSRs. It should be noted that this is an approach used in pharmacovigilance to assess the reporting frequency of
ICSRs within a drug class [14, 23, 24]. However, this type of analysis is not useful to assess a class effect on toxicity but other studies with different methodologies are needed on this front.

However, in addition to studies describing cases of myocardial fibrosis, pericarditis, and Takotsubo cardiomyopathy with ipilimumab [25-27], many clinical studies highlighted the occurrence of disparate cardiac ADRs during nivolumab and pembrolizumab treatments [28, 29]. Acute coronary syndrome, new-onset heart failure, and chronic heart failure have been also reported [29-31]. Interestingly, we also observed an increased reporting of ICI-related myocardial infarction, which underlying pathophysiology is currently unknown. However, three possible mechanisms have been hypothesized. First, the activation of ICI-related inflammation may induce the disruption of atherosclerotic coronary plaques and lead to acute myocardial infarction. Second, ICIs may cause coronary spasm causing ST elevation, which may also be related to the systemic inflammation induced by ICIs. Third, ICIs may directly activate the T-cell-mediated coronary vasculitis in the absence of atherosclerosis [32]. Further studies are needed to support these interesting pathophysiological hypotheses. Last, in our study, cardiovascular and metabolic agents (including calcium channel blockers, 
Table 5 Cardiovascular and metabolic agents reported as other suspected drugs in the individual case safety reports

\begin{tabular}{llc}
\hline Other suspected drugs & Number & Percentage \\
\hline Calcium channel blockers & 10 & 12.8 \\
New oral anticoagulants & 10 & 12.8 \\
Statins & 9 & 11.5 \\
Beta-blockers & 9 & 11.5 \\
Loop diuretics & 7 & 9.0 \\
Class III anti-arrhythmic agents & 5 & 6.4 \\
Injectable anticoagulants & 5 & 6.4 \\
Antiplatelets & 5 & 6.4 \\
Angiotensin receptor blockers & 4 & 5.1 \\
Potassium-sparing diuretics & 3 & 3.8 \\
Biguanide & 2 & 2.6 \\
Class Ic anti-arrhythmic agents & 1 & 1.3 \\
ACE inhibitors & 1 & 1.3 \\
Dipeptidyl peptidase-4 inhibitors & 1 & 1.3 \\
Fibrates & 1 & 1.3 \\
Insulins & 1 & 1.3 \\
Thiazide diuretics/angiotensin receptor & 1 & 1.3 \\
blockers & & 1.3 \\
Thiazide diuretics/beta-blockers & 1 & 1.3 \\
Thiazide diuretics/ACE inhibitors & 1 & 1.3 \\
Beta-blockers/antiplatelets & 1 & \\
Total & 78 & \\
\hline PCE & &
\end{tabular}

$A C E$ angiotensin-converting enzyme

Table 6 Cardiovascular and metabolic agents reported as concomitant drugs in the individual case safety reports

\begin{tabular}{llc}
\hline Concomitant drugs & Number & Percentage \\
\hline Beta-blockers & 172 & 12.9 \\
Calcium channel blockers & 145 & 10.9 \\
Antiplatelet agents & 142 & 10.7 \\
Statins & 139 & 10.5 \\
Angiotensin receptor blockers & 92 & 6.9 \\
Loop diuretics & 89 & 6.7 \\
ACE inhibitors & 81 & 6.1 \\
Insulins & 62 & 4.7 \\
New oral anticoagulants & 60 & 4.5 \\
Other agents & 348 & 26.2 \\
Total & 1330 & 100.0 \\
\hline
\end{tabular}

$A C E$ angiotensin-converting enzyme

new oral anticoagulants, statins, beta-blockers, and loop diuretics) were reported in $2.3 \%$ and $21.4 \%$ of all ICSRs among suspected and concomitant drugs, respectively. As these drugs are used in patients with cardiovascular diseases, the role of pre-existing diseases affecting the cardiovascular system on the occurrence of cardiac ADRs could not be excluded. Indeed, a retrospective matched case-control study, evaluating 108 cases of ICI-related myocarditis and 108 controls of ICI-related ADRs other than myocarditis obtained from VigiBase, found that drugs labeled as treatment for cardiovascular conditions were more strongly reported with ICI-related myocarditis than other ICI-related ADRs. In this study, compared to our result, a slightly increased percentage $(27.8 \%)$ of drugs belonging to the ATC "Cardiovascular system" was found among cases of ICI-related myocarditis. This higher percentage may be related to the different study design and to the different sample size [33]. In addition, patients diagnosed with cancer are more likely to die from cardiovascular diseases. Given this possible association, a closer cooperation between cardiologists and oncologists is strongly recommended [34].

In our study, the median time to event had a range of 22-36 days, suggesting the early occurrence of cardiotoxicity. In line with our results, literature data reported that the time to event for ICI-induced cardiac ADRs ranges from 12 days to 3 months [35].

\section{Strengths and Limitations}

A descriptive and statistical analysis has been carried out on data derived from the EV database. The spontaneous reporting system represents a useful and inexpensive tool for the collection and analysis of medicine safety data and for the better characterization of drug safety profiles. Indeed, using data from the spontaneous reporting system, specific ADRs, not detectable during the pre-marketing phase, including rare and serious ADRs, can be easily identified. Furthermore, the spontaneous reporting system involves ICSRs related to a frail population, which are usually excluded by the premarketing clinical trials. Therefore, through the EV database, we have analyzed a huge amount of ICSRs in which at least one ICI was reported as suspected. Furthermore, given the recent authorization of ICIs for the use in clinical practice and the consequent lack of data related to their safety profiles, we were able to provide information on the cardiotoxicity of these drugs that can be useful for oncologists.

However, the spontaneous reporting system carries some intrinsic limitations, such as the under-reporting and the poor quality of information listed in each ICSR. As we used EV data obtained from the EMA website (www.adrreports. $\mathrm{eu}$ ), we could only retrieve the information on the duration of therapy reported in days, the information on the number of ICSRs belonging to the SOC "cardiac disorders", and the information on single ICIs, differently from other studies using a different data source $[8,36]$. Therefore, we are aware that the real cardiac safety profile of ICIs cannot be fully established, but needs to be confirmed by the results 
Fig. 2 Reporting odds ratio (ROR) of individual case safety reports with cardiac adverse events for each immune checkpoint inhibitor (ICI) compared to all other ICIs. CI confidence interval
ICI

Nivolumab

Pembrolizumab

Atezolizumab

Durvalumab

Avelumab

Cemiplimab
Ipilimumab
Reference group

All other $\mathrm{ICls}$

All other $\mathrm{ICls}$

All other ICls

All other $\mathrm{ICls}$

All other ICls

All other $\mathrm{ICls}$

All other $\mathrm{ICls}$
ROR

$95 \% \mathrm{Cl}$

1.09

$1.01-1.18$

1.08

$0.99-1.17$

0.75

$0.68-0.84$

$0.91-1.32$

$0.68-1.05$

$0.88-2.01$

$0.28-1.71$ p-value

0.029

0.071

$<0.001$

0.334

0.133

0.177

0.429

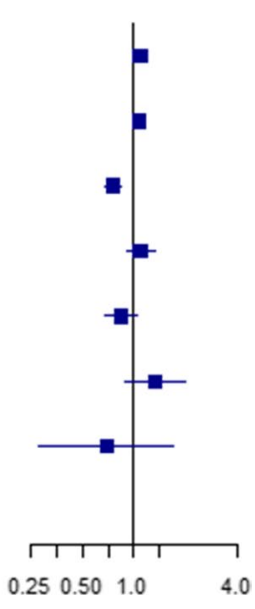

ICI

Nivolumab

Pembrolizumab

Cemiplimab

Atezolizumab

Durvalumab

Avelumab

\section{Reference group}

$$
\text { All other anti-PD-1 }
$$

0.99

All other anti-PD-1

1.01

$0.92-1.11$

1.00

$0.94-1.08$

1.18

$0.91-1.54$

All other anti-PD-L1

All other anti-PD-L1

0.75

0.57-0.99

All other anti-PD-L1 1.36

$0.88-2.10$

p-value

0.891

0.805

0.879

0.212

0.044

0.167
$0.91-1.09$

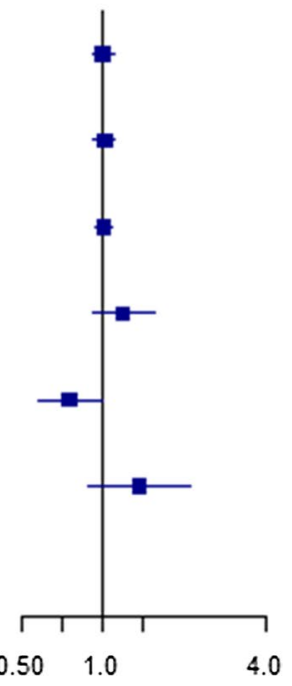

Fig. 3 Reporting odds ratio (ROR) of individual case safety reports with cardiac adverse events for each immune checkpoint inhibitor (ICI) compared to all other ICIs belonging to the same drug class. $C I$

confidence interval, $P D-1$ programmed cell death protein $1, P D-L 1$ programmed death-ligand 1
Fig. 4 Reporting odds ratio (ROR) of individual case safety reports with cardiac adverse events for anti-cytotoxic T-lymphocyte-associated antigen 4 (CTLA-4) compared to antiprogrammed cell death protein 1 (PD-1) and anti-programmed death-ligand 1 (PD-L1). $C I$ confidence interval

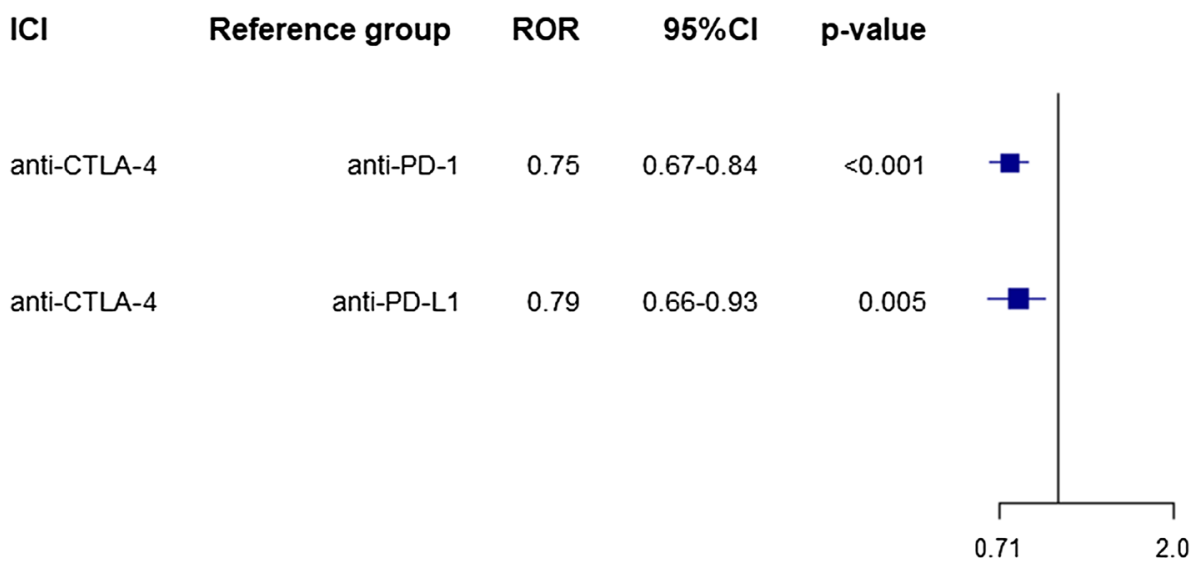


obtained from ad hoc studies. Furthermore, a limited number of ICSRs related to many ICIs and their combinations was found in the EV database. In addition, as we reported in our results, more than $20 \%$ of ICSRs reported concomitant medications, whose therapeutic indications were cardiovascular diseases. Their role in the occurrence of cardiac ADRs should be taken into consideration when the results of this study are evaluated.

\section{Conclusions}

We carried out descriptive and statistical analyses of data from 2478 ICSRs (covering 3388 cardiac ADRs) related to ICIs and cardiac ADRs. The majority of these ICSRs reported nivolumab and pembrolizumab as suspected drugs. The median age of patients who experienced cardiac ADRs was 69 years and most ICSRs referred to male patients. Cardiac ADRs were serious in more than $99 \%$ of cases and their outcome was fatal in $30.1 \%$ of cases. The most reported cardiac adverse events were myocarditis, cardiac failure, atrial fibrillation, pericardial effusion, and myocardial infarction. Applying the ROR, we found that nivolumab had a small increased reporting frequency of ICSRs with cardiac ADRs when compared to all other ICIs.

Immune checkpoint inhibitor-induced cardiac adverse effects are still underestimated. Given the seriousness of ICI-induced cardiac irADRs, routine clinical and laboratory assessments, such as repeat echocardiograms, cardiac biomarkers, and cardiac magnetic resonance imaging in the initial and later phases of ICI therapy, are strongly recommended. To provide new guidelines for oncologists and cardiologists for the management of these effects, a better understanding of the toxicity profiles of ICIs is strongly needed. Furthermore, clinicians should pay attention to possible predisposing factors of cardiac ADRs, including already existing cardiovascular diseases. In conclusion, given the potential seriousness of ICIinduced cardiac ADRs (in our study, almost $100 \%$ of ICSRs reported a serious ADR with a fatal outcome in $30 \%$ of cases) and considering the intrinsic limitations of our study as well as the recent marketing authorization of some ICIs, we believe that further high-quality clinical studies should be conducted on this topic to better estimate the impact of ICI therapy on cardiac safety in patients with cancer.

Supplementary Information The online version contains supplementary material available at https://doi.org/10.1007/s40264-021-01086-8.

\section{Declarations}

Funding Open access funding provided by Universit Ã degli Studi della Campania Luigi Vanvitelli within the CRUI-CARE Agreement. This research was funded under Grant no. 2017NR7W5K_002 (PRIN 2017) from MIUR, Italy. All authors had full access to all the data in the study and accepted responsibility to submit for publication.

Conflict of interest Marzia Del Re: Ipsen, Novartis, Pfizer, Sanofi Genzyme, AstraZeneca, Pierre-Fabre, and Janssen (scientific advisory board, consulting relationship) and Ipsen, AstraZeneca, and Sanofi Genzyme (travel, accommodation, expenses). Romano Danesi: Ipsen, Novartis, Pfizer, Sanofi Genzyme, AstraZeneca, Janssen, Gilead, Lilly, Gilead, and EUSA Pharma (scientific advisory board, consulting relationship) and Ipsen and Sanofi Genzyme (travel, accommodation, expenses). Annamaria Mascolo, Cristina Scavone, Carmen Ferrajolo, Concetta Rafaniello, Antonio Russo, Enrico Coscioni, Francesco Rossi, Roberto Alfano, and Annalisa Capuano have no conflicts of interest that are directly relevant to the content of this article.

Ethics approval Safety data deriving from the spontaneous reporting system are anonymous and comply with ethical standards. Therefore, no further ethical measure was required.

Consent to participate Not applicable.

Consent for publication Not applicable.

Availability of data and material The datasets generated for this study will not be made publicly available but European pharmacovigilance data are available at www.adrreports.eu.

Code availability Not applicable.

Authors' contributions AM, CS, RA, and AC wrote the original draft. $\mathrm{AM}, \mathrm{CS}, \mathrm{CF}, \mathrm{CR}, \mathrm{RD}, \mathrm{MDR}, \mathrm{AR}, \mathrm{EC}, \mathrm{FR}, \mathrm{RA}$, and AC were responsible for the conceptualization, data curation, methodology, project administration, and the writing, review, and editing of the manuscript. $\mathrm{AM}, \mathrm{CS}, \mathrm{CF}, \mathrm{CR}, \mathrm{RD}, \mathrm{MDR}, \mathrm{AR}, \mathrm{EC}, \mathrm{FR}, \mathrm{RA}$, and AC were responsible for resources and investigation. All authors approved the final version of the manuscript.

Open Access This article is licensed under a Creative Commons Attribution-NonCommercial 4.0 International License, which permits any non-commercial use, sharing, adaptation, distribution and reproduction in any medium or format, as long as you give appropriate credit to the original author(s) and the source, provide a link to the Creative Commons licence, and indicate if changes were made. The images or other third party material in this article are included in the article's Creative Commons licence, unless indicated otherwise in a credit line to the material. If material is not included in the article's Creative Commons licence and your intended use is not permitted by statutory regulation or exceeds the permitted use, you will need to obtain permission directly from the copyright holder. To view a copy of this licence, visit http://creativecommons.org/licenses/by-nc/4.0/.

\section{References}

1. Teixidó C, Vilariño N, Reyes R, Reguart N. PD-L1 expression testing in non-small cell lung cancer. Ther Adv Med Oncol. 2018;10:1758835918763493.

2. Koster BD, De Gruijl TD, Van Den Eertwegh AJM. Recent developments and future challenges in immune checkpoint inhibitory cancer treatment. Curr Opin Oncol. 2015;27:482-8.

3. Guan M, Zhou Y-P, Sun J-L, Chen S-C. Adverse events of monoclonal antibodies used for cancer therapy. Biomed Res Int. 2015;2015:428169. 
4. Brahmer JR, Lacchetti C, Schneider BJ, Atkins MB, Brassil KJ, Caterino JM, et al. Management of immune-related adverse events in patients treated with immune checkpoint inhibitor therapy: American Society of Clinical Oncology clinical practice guideline. J Clin Oncol. 2018;36:1714-68.

5. Michot JM, Bigenwald C, Champiat S, Collins M, Carbonnel F, Postel-Vinay S, et al. Immune-related adverse events with immune checkpoint blockade: a comprehensive review. Eur J Cancer. 2016;54:139-48.

6. Michel L, Rassaf T, Totzeck M. Cardiotoxicity from immune checkpoint inhibitors. Int J Cardiol Heart Vasc. 2019;25:100420.

7. Upadhrasta S, Elias H, Patel K, Zheng L. Managing cardiotoxicity associated with immune checkpoint inhibitors. Chronic Dis Transl Med. 2019;5:6-14.

8. Salem JE, Manouchehri A, Moey M, Lebrun-Vignes B, Bastarache L, Pariente A, et al. Cardiovascular toxicities associated with immune checkpoint inhibitors: an observational, retrospective, pharmacovigilance study. Lancet Oncol. 2018;19:1579-89.

9. Garon-Czmil J, Petitpain N, Rouby F, Sassier M, Babai S, YéléhéOkouma M, et al. Immune check point inhibitors-induced hypophysitis: a retrospective analysis of the French pharmacovigilance database. Sci Rep. 2019;9:1-5.

10. Shivaji UN, Jeffery L, Gui X, Smith SCLL, Ahmad OF, Akbar A, et al. Immune checkpoint inhibitor-associated gastrointestinal and hepatic adverse events and their management. Ther Adv Gastroenterol. 2019;12:175628481988419.

11. Matthews NH, Li WQ, Qureshi AA, Weinstock MA, Cho E. Epidemiology of melanoma. In: Ward WH, Farma JM, editors. Cutaneous melanoma: etiology and therapy. Brisbane: Codon Publications; 2017.

12. Capitanio U, Bensalah K, Bex A, Boorjian SA, Bray F, Coleman J, et al. Epidemiology of renal cell carcinoma. Eur Urol. 2019;75:74-84.

13. de Groot PM, Wu CC, Carter BW, Munden RF. The epidemiology of lung cancer. Transl Lung Cancer Res. 2018;7:220-33.

14. Ruggiero R, Fraenza F, Scavone C, di Mauro G, Piscitelli R, Mascolo A, et al. Immune checkpoint inhibitors and immune-related adverse drug reactions: data from Italian pharmacovigilance database. Front Pharmacol. 2020;11:830.

15. Rotte A. Combination of CTLA-4 and PD-1 blockers for treatment of cancer. J Exp Clin Cancer Res. 2019;38:1-12.

16. Scavone C, Sportiello L, Sullo MG, Ferrajolo C, Ruggiero R, Sessa $\mathrm{M}$, et al. Safety profile of anticancer and immune-modulating biotech drugs used in a real world setting in Campania Region (Italy): BIO-Cam observational study. Front Pharmacol. 2017;8:607.

17. Wang DY, Salem JE, Cohen JV, Chandra S, Menzer C, Ye F, et al. Fatal toxic effects associated with immune checkpoint inhibitors: a systematic review and meta-analysis. JAMA Oncol. 2018;4:1721-8.

18. Nishimura H, Okazaki T, Tanaka Y, Nakatani K, Hara M, Matsumori A, et al. Autoimmune dilated cardiomyopathy in PD-1 receptor-deficient mice. Science. 2001;291:319-22.

19. Zhou YW, Zhu YJ, Wang MN, Xie Y, Chen CY, Zhang T, et al. Immune checkpoint inhibitor-associated cardiotoxicity: current understanding on its mechanism, diagnosis and management. Front Pharmacol. 2019;10:1350.

20. Stein-Merlob AF, Rothberg MV, Holman P, Yang EH. Immunotherapy-associated cardiotoxicity of immune checkpoint inhibitors and chimeric antigen receptor $\mathrm{T}$ cell therapy: diagnostic and management challenges and strategies. Curr Cardiol Rep. 2021;23:1-11.

21. Pirozzi F, Poto R, Aran L, Cuomo A, Galdiero MR, Spadaro G, et al. Cardiovascular toxicity of immune checkpoint inhibitors: clinical risk factors. Curr Oncol Rep. 2021;23:1-8.
22. Balanescu DV, Donisan T, Palaskas NL, Iliescu C. Emerging concerns in cardio-oncology: immune checkpoint inhibitor cardiotoxicity. Am Coll Cardiol. 2019;4-8. https://www.acc.org/ latest-in-cardiology/articles/2019/06/21/08/45/emerging-conce rns-in-cardio-oncology.

23. Noseda R, Bonaldo G, Motola D, Stathis A, Ceschi A. Adverse event reporting with immune checkpoint inhibitors in older patients: age subgroup disproportionality analysis in VigiBase. Cancers (Basel). 2021;13:1131.

24. Raschi E, Mazzarella A, Antonazzo IC, Bendinelli N, Forcesi E, Tuccori M, et al. Toxicities with immune checkpoint inhibitors: emerging priorities from disproportionality analysis of the FDA Adverse Event Reporting System. Target Oncol. 2019;14:205-21.

25. Yun S, Vincelette ND, Mansour I, Hariri D, Moramed S. Late onset ipilimumab-induced pericarditis and pericardial effusion: a rare but life threatening complication. Case Rep Oncol Med. 2015;2015:5.

26. Geisler BP, Raad RA, Esaian D, Sharon E, Schwartz DR. Apical ballooning and cardiomyopathy in a melanoma patient treated with ipilimumab: a case of takotsubo-like syndrome. J Immunother Cancer. 2015;3:4.

27. Voskens CJ, Goldinger SM, Loquai C, Robert C, Kaehler KC, Berking $\mathrm{C}$, et al. The price of tumor control: an analysis of rare side effects of anti-CTLA-4 therapy in metastatic melanoma from the ipilimumab network. PLoS ONE. 2013;8:e53745.

28. Gibson R, Delaune J, Szady A, Markham M. Suspected autoimmune myocarditis and cardiac conduction abnormalities with nivolumab therapy for non-small cell lung cancer. BMJ Case Rep. 2016;2016:bcr2016216228.

29. Tadokoro T, Keshino E, Makiyama A, Sasaguri T, Ohshima K, Katano H, et al. Acute lymphocytic myocarditis with anti-PD-1 antibody nivolumab. Circ Heart Fail. 2016;9:e003514.

30. Läubli H, Balmelli C, Bossard M, Pfister O, Glatz K, Zippelius A. Acute heart failure due to autoimmune myocarditis under pembrolizumab treatment for metastatic melanoma. J Immunother Cancer. 2015;3:11.

31. Caforio AL, Pankuweit S, Arbustini E, Basso C, Gimeno-Blanes J, Felix SB, et al. Current state of knowledge on aetiology, diagnosis, management, and therapy of myocarditis: a position statement of the European Society of Cardiology Working Group on Myocardial and Pericardial Diseases. Eur Heart J. 2013;34:2636-48.

32. Chen DY, Huang WK, Chien-Chia WuV, Chang WC, Chen JS, Chuang CK, et al. Cardiovascular toxicity of immune checkpoint inhibitors in cancer patients: a review when cardiology meets immuno-oncology. J Formos Med Assoc. 2020;119:1461-75.

33. Noseda R, Ruinelli L, van der Gaag LC, Ceschi A. Pre-existing cardiovascular conditions as clinical predictors of myocarditis reporting with immune checkpoint inhibitors: a VigiBase study. Cancers (Basel). 2020;12:3480.

34. Sturgeon KM, Deng L, Bluethmann SM, Zhou S, Trifiletti DM, Jiang C, et al. A population-based study of cardiovascular disease mortality risk in US cancer patients. Eur Heart J. 2019;40:3889-97.

35. Johnson DB, Balko JM, Compton ML, Chalkias S, Gorham J, $\mathrm{Xu} \mathrm{Y}$, et al. Fulminant myocarditis with combination immune checkpoint blockade. N Engl J Med. 2016;375:1749-55.

36. Fan Q, Hu Y, Yang C, Zhao B. Myocarditis following the use of different immune checkpoint inhibitor regimens: a real-world analysis of post-marketing surveillance data. Int Immunopharmacol. 2019;76:105866.

37. Freeman GJ, Long AJ, Iwai Y, et al. Engagement of the PD-1 immunoinhibitory receptor by a novel B7 family member leads to negative regulation of lymphocyte activation. J Exp Med. 2000;192(7):1027-34. https://doi.org/10.1084/jem.192.7.1027. 


\section{Authors and Affiliations}

\section{Annamaria Mascolo ${ }^{1,2} \cdot$ Cristina Scavone ${ }^{1,2} \cdot$ Carmen Ferrajolo $^{1,2} \cdot$ Concetta Rafaniello $^{1,2} \cdot$ Romano Danesi $^{3}$.} Marzia Del $\mathrm{Re}^{3} \cdot$ Antonio Russo $^{4}$ - Enrico Coscioni ${ }^{5}$. Francesco Rossi ${ }^{1,2} \cdot$ Roberto Alfano $^{6}$ - Annalisa Capuano ${ }^{1,2}$

1 Campania Regional Centre for Pharmacovigilance and Pharmacoepidemiology, Naples, Italy

2 Department of Experimental Medicine, Section of Pharmacology, "L. Donatelli", University of Campania "Luigi Vanvitelli", Naples, Italy

3 Unit of Clinical Pharmacology and Pharmacogenetics, Department of Clinical and Experimental Medicine, University Hospital of Pisa, Pisa, Italy
4 Department of Oncology, A.O.U.P. "P. Giaccone”, University Hospital Palermo, Palermo, Italy

5 AGENAS-Agenzia Nazionale per i Servizi Sanitari Regionali, Rome, Italy

6 Unità Operativa Semplice Interdipartimentale Programmazione e Coordinamento Attività Sanitaria, Azienda Ospedaliera Universitaria, University of Campania "Luigi Vanvitelli", Naples, Italy 